\title{
MICHEL ALBOUY
}

Grenoble école de Management ; Université Grenoble Alpes

\section{Alerte sur l'enseignement supérieur de gestion français ${ }^{1}$}

$\mathrm{L}^{\prime}$

Tenseignement supérieur de gestion des entreprises est aujourd'hui en difficulté et court prochainement à sa perte si les pouvoirs publics n'en prennent pas conscience. Tous les efforts qui ont été accomplis depuis la création de la Fondation nationale pour l'enseignement de la gestion des entreprises (Fnege) créée en 1968 par l'État français et le secteur privé dans le but de développer un véritable enseignement supérieur de gestion risquent d'être perdus.

Rappelons-nous : il y a seulement 50 ans, il n'existait pas véritablement de corps professoral de sciences de gestion en France. Certes, il y avait bien des écoles de commerce qui dispensaient des formations appliquées, mais elles faisaient essentiellement appel à des professionnels d'entreprises et quelques professeurs de droit et d'économie des facultés. Personne

1. Une première version de cet article a été publiée dans The Conversation, 21 septembre 2017. Cette version intègre certains commentaires faits par les lecteurs de ce site. Nous remercions tout particulièrement Jean-Pierre Nioche pour ses remarques qui nous ont permis d'améliorer le texte original. Cependant, nous avons souhaité conserver la tonalité de l'article original qui avait essentiellement pour vocation à « tirer une sonnette d'alarme » et non à produire une recherche académique dans les règles de l'art. Cet article s'appuie cependant sur notre longue expérience acquise dans les deux systèmes de l'enseignement supérieur de gestion. 
pour faire des manuels de gestion, personne pour rédiger des études de cas, pas de revues académique dans cette discipline qui était à inventer. C'est avec la création de la Fnege et son programme phare de formation aux États-Unis d'enseignants de gestion que la dynamique s'est enclenchée. Ce programme d'envoi de jeunes boursiers aux États-Unis a permis de former le socle des enseignants-chercheurs en gestion qui manquait tant à la France. À leur retour, les boursiers de la Fnege - souvent titulaires d'un doctorat américain ( $\mathrm{PhD})$ - ont intégré les universités (parfois difficilement) et les grandes écoles de commerce qui n'avaient à cette époque qu'un seul programme: le diplôme de l'école. Ce n'est seulement qu'en 1976 - il y a un peu plus de 40 ans seulement - que l'université française a créé l'agrégation des sciences de gestion pour le recrutement de professeurs des universités dans cette discipline. Avant cela, il n'y avait pas de carrière pour les enseignants-chercheurs en gestion à l'université. Ils restaient maître de conférences, plus ou moins tolérés par les professeurs de sciences économiques qui eux-mêmes s'étaient libérés, peu d'années avant, des professeurs de droit. En 1975 la première revue consacrée aux sciences de gestion était lancée : la Revue française de gestion. Suivront par la suite, sous l'impulsion des associations académiques, beaucoup d'autres revues plus spécialisées selon les différents champs disciplinaires des sciences de gestion : finance, marketing, contrôle, management stratégique, gestion des ressources humaines, etc. Bref, la gestion acquérait ses lettres de noblesse académiques et se voyait reconnaître comme discipline à part entière à l'université.
Aujourd'hui, 50 ans après la création de la Fnege, on ne peut que se féliciter de cette réussite. Nos grandes écoles de management caracolent dans les classements du Financial Times et les Instituts d'administration des entreprises (IAE) permettent au plus grand nombre d'accéder à des formations professionnelles de haut niveau en gestion des entreprises (Master). Ajoutons au tableau, et cela dans la suite de Mai 1968, la création de l'université Paris-Dauphine qui doit sa réussite à son caractère de Grand établissement.

Pourtant cette réussite est aujourd'hui fragile et il ne faudrait pas croire que notre système d'enseignement supérieur en sciences de gestion connaîtra à coup sûr la même trajectoire de réussite dans le futur. En effet, plusieurs défis se dressent sur la route de nos écoles et universités. Le premier défi est celui de la mondialisation des formations en management. Le second défi est celui de leur vrai manque de moyens financiers pour appréhender cette concurrence. Face à ces défis, les deux systèmes de l'enseignement supérieur français de gestion n'ont pas les même atouts ni les même contraintes, ils restent très fragiles. Prenons-les successivement.

\section{Les dilemmes des grandes écoles de commerce : le manque d'argent et la concurrence internationale}

Tout d'abord les grandes écoles de management (qui se font aussi appeler business schools). Ces écoles, grâce à leur indépendance et leur liberté de fonctionnement, ont pu véritablement s'internationaliser depuis les années 2000 (c'est relativement récent). Elles ont noué des partenariats avec de très nombreuses universités étrangères (plus ou moins bonnes) et permettent à leurs 
étudiants d'aller aux quatre coins du monde. Elles ont aussi développé de nombreux programmes internationaux (MBA, MIB, MSc notamment) qui leur permettent de recruter de nombreux étudiants étrangers. Elles se sont également lancées dans la recherche académique et ont créé des programmes doctoraux (PhD et DBA). Pour cela elles ont aussi recruté massivement des professeurs étrangers de haut niveau, ce qui ne va pas toujours sans poser de problèmes. Malgré l'augmentation considérable du nombre de leurs diplômés elles continuent à les intégrer au monde du travail malgré la concurrence nationale des IAE. Jusqu'à présent, leurs places dans les classements internationaux (ranking) et notamment ceux du célèbre Financial Times étaient exceptionnelles. Par exemple, HEC Paris a été pendant de très nombreuses années classée $\mathrm{n}^{\circ} 1$ par le Financial Times (FT) devant la célèbre London School of Business. Nombreuses sont nos grandes écoles de commerce à figurer dans le top 20 du Financial Times. Mais aujourd'hui, et devant la concurrence mondiale, elles commencent à reculer. Légèrement pour le moment, mais cela risque de s'aggraver comme le souligne l'ancien directeur général d'HEC Bernard Ramanantsoa (cf. Xerfi Canal, 18/09/2017) qui pointe le décrochage récent de nos écoles de management.

Le tableau 1 montre la perte de compétitivité de nos grandes écoles de management selon le classement des masters du Financial Times. Comme on peut le constater, toutes (sauf HEC Paris) perdent des places. Et cela au profit de nouveaux entrants sur le marché de la formation en gestion des entreprises.
Tableau 1 - Classement international des masters de management des écoles françaises par le Financial Times

\begin{tabular}{|l|c|c|c|}
\hline \multicolumn{1}{|c|}{ Rang } & $\mathbf{2 0 1 6}$ & $\mathbf{2 0 1 7}$ & Changement \\
\hline HEC Paris & 2 & 2 & 0 \\
\hline ESSEC & 3 & 5 & -2 \\
\hline $\begin{array}{l}\text { ESCP } \\
\text { Europe }\end{array}$ & 4 & 6 & -2 \\
\hline EDHEC & 15 & 16 & -1 \\
\hline EM Lyon & 26 & 27 & -1 \\
\hline Audencia & 24 & 29 & -5 \\
\hline IESEG & 17 & 31 & -14 \\
\hline $\begin{array}{l}\text { Grenoble } \\
\text { EM }\end{array}$ & 13 & 33 & -20 \\
\hline Skema & 26 & 35 & -9 \\
\hline Neoma & 34 & 40 & -6 \\
\hline
\end{tabular}

Source : Financial Times.

Aujourd'hui la scolarité d'un élève de programme « grande école de commerce » tourne autour de 35000 euros pour les trois années d'études, parfois davantage. À ce montant il faut bien évidemment ajouter les frais d'hébergement et de transport, soit 6000 euros/an au bas mot. Au total cela fait donc au minimum pour un diplôme d'école de commerce 53000 euros hors frais de bouche. C'est cher payé pour un diplôme, qui, sauf pour les trois plus grandes (HEC, ESSEC, ESCP), ne fera pas une grosse différence avec de bons Master d'IAE (qu'on peut trouver à côté de chez soi), sans parler de l'université Paris-Dauphine. La comparaison - souvent évoquée par les directeurs des business schools françaises avec les tarifs pratiqués avec les grandes 
universités américaines n'a pas grand sens. Outre le fait que ces grandes universités américaines offrent un tout autre environnement, les parents américains sont très loin de supporter les prélèvements fiscaux de nos compatriotes qui payent également avec leurs impôts pour le fonctionnement des universités. De plus, même les universités publiques américaines sont payantes, contrairement aux universités françaises. Il n'existe donc pas aux États-Unis l'équivalent des IAE qui sont quasiment gratuits pour des formations supérieures en gestion. L'arbitrage est donc différent. Et cela sans compter avec l'existence d'une université publique comme Dauphine qui revendique également, et à juste titre, l'excellence en recherche comme en formation.

Pour jouer dans la cour des grands - c'est-àdire des business schools internationales du top 30 - nos grandes écoles de commerce ont dû recruter à grand frais des enseignantschercheurs internationaux (disons plutôt des chercheurs tant leur implication pédagogique reste faible) pour progresser dans les fameux rankings. Face à l'explosion de leurs coûts de fonctionnement, elles ont fondamentalement joué sur deux leviers : la hausse des frais de scolarité et l'augmentation du nombre d'étudiants. La hausse des frais de scolarité a atteint aujourd'hui un plafond.

Difficile de penser que dans les dix prochaines années, les augmentations enregistrées à ce jour pourront se perpétuer, surtout dans un environnement économique à inflation quasi nulle. On arrive ainsi à un palier et les marges de manœuvres sont très limitées sinon inexistantes dans ce domaine. En effet, et contrairement à ce que d'aucuns croient, ce n'est pas en faisant de la formation continue ou du conseil auprès des entreprises que ces écoles pourront équilibrer leur budget qui est en fait souvent financé à plus de $85 \%$, voire $90 \%$, par les étudiants; les chambres de commerce s'étant retirées. Les étudiants se retrouvent donc à financer la recherche de professeurs qui pourront aller ailleurs au grès du « mercato » international des professeurs de gestion. De plus, vu la charge d'enseignement relativement faible de ces supers stars de la recherche, ils ont peu de chances de les avoir comme enseignant. Quant à l'augmentation des effectifs étudiants, on a également atteint un niveau qu'il sera difficile de repousser, sauf à perdre ce qui fait l'essence même d'une école : des promotions à taille humaine. Dans ces conditions comment continuer à recruter davantage d'enseignants-chercheurs capables de publier dans des revues de premier rang ? Comment rester dans la course mondialisée aux étoiles CNRS (référence au classement de la section 37 économiegestion du CNRS) ? Admettre qu'on n'est plus qu'une « teaching institution »? Mais dans ces conditions comment continuer à recruter des étudiants internationaux pour des masters (MSc) à 30000 euros ? L'équation semble sans solution. Et c'est bien là le drame des grandes écoles françaises de gestion qui ne peuvent compter sur aucune aide de l'État alors qu'elles contribuent à la compétitivité des entreprises françaises et au rayonnement de l'enseignement supérieur français.

Parmi les voies pour associer davantage les entreprises, mais également les collectivités locales, se trouve le nouveau statut d'EESC (Établissement d'enseignement supérieur consulaire) créé fin 2014 par la loi Mandon qui permet aux écoles de commerce de devenir autonomes sur le plan juridique. 
Outre une autonomie et une souplesse de gestion proche du fonctionnement d'une société anonyme à but non lucratif, le statut d'EESC devrait permettre aux écoles de diversifier leurs sources de financement en accueillant au sein de leur capital des acteurs privés et publics. En créant une EESC, la CCI (chambre de commerce) dote l'école d'un capital constitué des actifs qu'elle aura transféré comme par exemple les moyens d'exploitation, la marque, voire même l'immobilier et le foncier pour les mieux pourvues. Pour le moment les grandes écoles de gestion passées au statut EESC sont : HEC Paris (pour qui le statut a été initialement conçu), Grenoble École de management (GEM), Toulouse Business school (TBS) et l'ESC Dijon. Une vingtaine d'autres écoles, comme ESCP Europe et Audencia, devraient suivre. À noter cependant que selon les négociations entre les écoles et leur tutelle consulaire, la corbeille de la mariée a été plus ou moins garnie. Alors que pour certaines écoles le CCI a transféré le patrimoine immobilier il n'en va pas de même pour d'autres qui se trouvent obligées de verser un loyer à leur tutelle, ce qui n’arrange pas leurs finances.

\section{Les dilemmes des formations universi- taires : les contraintes et le manque de liberté}

Sauf à de rares exceptions près, comme l'université Paris-Dauphine, l'enseignement des sciences de gestion à l'université se trouve essentiellement dans les $\mathrm{IAE}^{2}$. Ces derniers réunis dans un réseau national, se présentent depuis 2014 comme des écoles universitaires de management. Le réseau compte 32 instituts et environ 45000 étudiants. Ils proposent plus de 30 parcours de la licence au doctorat en sciences de gestion. Leur présence sur le territoire est incontestablement une réussite au niveau franco-français. Cette forte présence, s'il elle est un atout, constitue également une faiblesse : quelle différentiation ? Pourquoi aller plutôt à Lyon qu'à Grenoble ou à Lille dans la mesure où il n'existe pas de classement ? Certes, le choix pourra se porter en fonction des spécialités offertes au niveau des masters 2, mais cela reste marginal vu la propension des étudiants des universités de rester sur leur territoire. Au niveau international, le paysage est plus contrasté. Globalement leur internationalisation est relativement modeste et leur reconnaissance internationale faible. Par exemple, à part l'IAE d'Aix en Provence (le premier IAE créé en 1955), aucun n'est titulaire de l'accréditation internationale EQUIS, alors que les grandes écoles de commerce (top 10) le sont toutes. Elles ont souvent aussi les trois couronnes internationales (AACSB, AMBA, EQUIS). Bien sûr, l'université Dauphine possède le label EQUIS mais elle est bien la seule université française dans ce cas.

Bien sûr les IAE, étant des composantes d'universités, bénéficient des avantages et des inconvénients de cette situation. Par exemple, ils n'ont pas à payer leurs professeurs contrairement aux grandes écoles car ce sont des fonctionnaires d'État.

2. Certains commentateurs m'ont fait remarquer à juste titre que la gestion à l'université n'était pas exclusivement enseignée dans les IAE. De fait, on retrouve dans de nombreux cursus universitaires de licence ou de master des enseignements de gestion. Cependant, les IAE constituent, à mon sens, la forme la plus achevée de création d'établissements universitaires dédiés spécifiquement à l'enseignement de la gestion. À ce titre ils sont porteurs d'un modèle bien spécifique et alternatif à celui des grandes écoles de commerce. 
Ils ne payent pas également leurs personnels administratifs (sauf à la marge). En échange ils n'ont aucune autonomie de gestion. Ils ne peuvent faire payer leurs étudiants (même étrangers), ils ne peuvent pas vraiment les sélectionner (comme les écoles) à travers des concours nationaux et surtout ils ne peuvent que très difficilement recruter des professeurs étrangers du fait des procédures spécifiques d'accès aux corps des professeurs d'universités. Bref, et ce n'est pas leur faire injure, leurs marges de manœuvre sont des plus réduites. Pas de quoi partir à la conquête du monde, sauf exception locale. Et pour quoi faire puisque dans tous les cas cela ne ferait que consommer des ressources contraintes allouées par l'État sans véritablement mettre en face des recettes nouvelles (absence de frais de scolarité). Or, sans internationalisation ces instituts ne pourront que s'étioler ou au mieux ne servir que leur territoire de proximité.

Par ailleurs, alors que pour exister au niveau international il faut absolument publier dans des revues internationales de premier rang, la composition très franco-française des corps enseignants des IAE rend difficile cette mutation. Il y a donc de fortes chances qu'à moyen terme ces instituts ne deviennent que des «teaching institutions » pour reprendre le vocabulaire de l'AACSB (Association américaine d'accréditation pour l'enseignement du management).

Alors que les IAE semblent bénéficier du confort protecteur ${ }^{3}$ des universités publiques, ils ne peuvent pas vraiment s'épanouir comme n'importe quelle business school américaine qui, même si elle appartient à un campus, bénéficie d'une véritable autonomie de gestion (sélection des étudiants, frais de scolarité, procédures de recrutement du corps enseignant, etc.). Ajoutons à cela qu'avec leur intégration (pour ne pas dire absorption) de plus en plus forte au sein des universités (éventuellement fusionnées), ces instituts ont perdu les quelques petites marges de manœuvre qu'ils avaient dans les années 1980 avec la formation continue. Enfin, le statut de la fonction publique pour des enseignants-chercheurs internationaux en management de bon niveau n'a rien d'attractif. Dans ces conditions avec ce maillage de contraintes, on peut vraiment s'interroger sur la future trajectoire de développement de ces instituts. On note du reste que de nombreux docteurs formés dans les universités rejoignent les grandes écoles (cf. l'étude de la Fnege par S. Point sur « l'insertion professionnelle des docteurs en sciences de gestion : entre mythes et réalités, 2013) et n'intègrent pas l'université. Leurs raisons sont diverses mais parmi elle se trouve l'écart de rémunération au moment du recrutement et les conditions de travail.

\section{Et pourtant l'enseignement supérieur de gestion est indispensable pour la France}

Le tableau que nous venons de dresser apparaîtra à certains comme volontairement noirci et excessif. Bien sûr, le système mis en place avec la création de la Fnege continue à fonctionner, et même bien. Mais pour combien de temps ? À notre avis mais chacun peut avoir le sien - pour pas

3. Ce qualificatif pourra sembler inapproprié aux enseignants-chercheurs des universités qui voient leurs conditions de travail se dégrader avec la réduction des dotations de l'État et la hausse des effectifs étudiants. Mais pour bien connaître les deux systèmes, il est clair que le statut protecteur de la fonction publique constitue un sérieux avantage par rapport à celui des écoles consulaires (EESC). Les systèmes de sanction/récompense sont également fort différents. 
très longtemps. Les grandes écoles de commerce vont faire face assez rapidement à des révisions difficiles. Quant aux formations universitaires, IAE compris, elles vont avoir du mal à rester dans la compétition mondiale qui s'annonce féroce avec l'émergence de la Chine et de l'Inde dans les formations au management.

Parmi les révisions à faire se trouve la place de la recherche dans les grandes écoles et les IAE. Est-ce que toutes ces institutions ont vocation à faire de la recherche de très haut niveau et à recruter des professeurs capables de publier dans des revues internationales de rang 1 ou 2 du classement de la section $37 \mathrm{du}$ CNRS ? On peut en douter ? Et puis, de quelle recherche parlons-nous ? Si la recherche en gestion s'est développée, grâce notamment à l'impulsion décisive de la Fnege, c'est principalement pour répondre à une meilleure connaissance des pratiques de gestion et/ou de management et participer à leur amélioration. Ce n'était pas pour augmenter le flux de recherche dans les disciplines fondamentales des sciences sociales comme l'économie, la sociologie ou les mathématiques. Or, en voulant publier dans les revues les plus prestigieuses de ces disciplines, on s'éloigne du caractère appliqué des sciences de gestion. Par exemple, dans le cas de la finance, les revues les plus cotées relèvent davantage de l' « économie financière » que de la « gestion financière » proprement dite. Les problématiques de gestion financière des entreprises sont ainsi reléguées dans des revues de moindre rang académique, ce qui éloigne les chercheurs qui veulent progresser dans leur carrière de ces problématiques gestionnaires plus appliquées. Ces revues moins étoilées rapportent également moins de prime à leurs auteurs. On pourrait en dire de même pour ce qui concerne la gestion des ressources humaines et les autres champs des sciences de gestion. La conséquence de ce comportement est que la production de recherche des écoles et des universités en gestion a tendance à s'éloigner des préoccupations des entreprises. Notre propre expérience de membre de jury du concours d'agrégation pour le recrutement des professeurs en sciences de gestion (2010) nous renforce dans cette vision. C'est ainsi que l'entreprise disparaît progressivement des radars des chercheurs : en finance on ne s'intéresse plus qu'au fonctionnement des marchés financiers, en marketing l'étude du comportement du consommateur a pris le pas sur le marketing industriel, etc. Les chercheurs ne vont plus dans les entreprises (cela prend trop de temps) mais utilisent des bases de données ou des enquêtes postales ou via Internet. Dans ces conditions, il ne faut pas s'étonner de la faible appétence des dirigeants et cadres d'entreprise pour la production de recherche en sciences de gestion. Loin de nous de rejeter ici l'intérêt des publications des revues académique de très haut niveau pour faire avancer les sciences fondamentales, mais est-ce bien la mission des écoles de commerce et des IAE que de vouloir aller sur le terrain des centres de recherche financés par le CNRS ? La question se pose et il faudra bien y répondre. Et puis, dans quelle mesure cette production de recherche permet de faire vraiment évoluer les enseignements ? Alors que dans les écoles d'ingénieurs la recherche irrigue les cours les plus avancés cela ne semble pas être le cas dans les écoles de management. Les formations en gestion des entreprises sont indispensables à la compétitivité de nos 
entreprises qui ont besoin de cadres bien formés pour se lancer à la conquête des marchés étrangers. La réussite de nos grandes entreprises à l'international est là pour montrer l'apport décisif de ces formations qui savent allier pragmatisme, rigueur et international. Il est donc urgent de prendre la mesure des difficultés que traverse notre système d'enseignement supérieur de gestion à l'heure actuelle, mais surtout pour les années futures. Il serait vraiment dommage que le sursaut fait au moment de la création de la Fnege il y a 50 ans se perde.

Alors que faire ? Au-delà des questions fondamentales que soulève la place de la recherche dans les établissements d'enseignement supérieur de gestion, nous avançons trois propositions :

1) Tout d'abord il faut que l'État, mais également les entreprises privées et publiques, injectent davantage d'argent dans nos écoles et universités. À eux de voir comment, mais il est clair que les écoles ne pourront pas continuer à demander à leurs professeurs et collaborateurs de toujours faire plus (d'étoiles CNRS et de cours) avec moins de ressources et à leurs étudiants de payer toujours plus avec des effectifs toujours plus nombreux. Globalement, et alors que l'insertion de leurs diplômés est remarquable, les sciences de gestion qui répondent aux besoins des entreprises sont sous-dotées, surtout si on les compare à d'autres disciplines comme par exemple les sciences économiques. Cette implication plus forte des entreprises serait susceptible de peser sur les programmes de recherche des écoles.
2) Il faudrait que les IAE retrouvent de véritables marges de manœuvres compte tenu des spécificités de l'enseignement supérieur de gestion. On ne pourra pas développer des instituts compétitifs dans cette discipline exposée à la concurrence mondiale avec des statuts universitaires qui empêchent toute initiative. En son temps, la loi Savary de 1984 sur l'enseignement supérieur avait créé le statut d'écoles universitaires (article 33) de façon à laisser plus d'autonomie aux instituts tels que les IAE. Malheureusement, et sous la pression des présidents d'universités et de leurs Conseils, ce statut n'a jamais été appliqué même pour les IAE qui avaient obtenu ce label par le Ministère. Bien au contraire, ils ont subi les effets de la centralisation et de la normalisation au motif de l'égalité de traitement des composantes de l'université. 3) Enfin, et c'est ma troisième proposition: il faut que les écoles et les universités collaborent davantage, notamment sur la recherche et la formation doctorale car dans quelques années la recherche française en sciences de gestion sera devenue inaudible au niveau mondial. Si cette dynamique semble se mettre en place sur Paris avec la participation des plus grandes écoles de management aux écoles doctorales, il n'en va pas de même en province. Ajoutons pour terminer que tout cela passe également par la capacité de nos programmes doctoraux en gestion d'attirer les meilleurs talents du monde (cf. notre étude avec A.C. Martinet pour la Fnege sur « les programmes doctoraux de gestion en France à l'heure de la mondialisation », septembre 2017). 